\title{
DIVERSIFICACIÓN Y CARTELIZACIÓN EN EL SEGURO ESPAÑOL, 1914-1935
}

\author{
JERÒNIA PONS PONS \\ Universidad de Sevilla
}

\section{RESUMEN}

En el primer tercio del siglo xx se producen varios fenómenos en el mercado de seguros en España por el que se modifica la situación heredada del siglo anterior. El incremento de la competencia impulsa procesos de diversificación y cartelización del mercado. En el primer caso-las compañías especializadas se convertirán en compañías generales y en el segundo caso se adoptaran acuerdos entre las empresas de seguros para controlar la competencia. En las décadas posteriores, estos fenómenos paralelos conducirán al liderazgo a determinadas compañías.

\begin{abstract}
In the first decades of the 20th century, several processes modify the Spanish insurance market from the inherited characteristics of the past century. Moreover, the increment of competition promotes the diversification and setting up cartels for control of market. The first process drives the specialised companies to general companies and the second one leads to agreements between them to control the competition. Both parallel procedures originate later on the leadership of particular companies.

JEL Classification: G22.

El desarrollo histórico del mercado de seguros contemporáneo en España es un tema escasamente investigado. Aparte de algún trabajo procedente del propio negocio asegurador (Maestro, 1991 y 1993) hay que esperar


al novedoso artículo de Frax y Matilla publicado en 1996 en la Revista de Historia Económica para que se aborde el tema desde el ámbito de la historia económica. Gracias a este trabajo conocemos algunas de las características del mercado español que se heredaron a principios del siglo $\mathrm{xx}$, tales como el mayor peso de los ramos tradicionales como el seguro de incendios o marítimo, el predominio de las compañías especializadas o la importancia de las compañías extranjeras. A esta investigación ambas autoras añadieron un enfoque comparativo con el seguro francés en 1998. Este árido panorama nos indujo a saltar desde el conocimiento del seguro moderno al estudio de la evolución del seguro contemporáneo, primero desde el ámbito regional (Pons, 1998) y a continuación profundizando en el mercado nacional durante el primer tercio del siglo xx (Pons, 2001, 2002a y 2002b).

En este artículo, gracias a la disponibilidad de estadísticas oficiales continuas a partir de la Ley de Seguros de 1908 -sobre todo tras la aprobación del Reglamento de 1912 - se aportan novedades sobre una serie de cambios que afectan al mercado español y a las estrategias de crecimiento de las compañías de seguro. En un primer apartado se estudia el mercado del seguro a principios de siglo proporcionando datos cuantitativos sobre la evolución y situación por ramos, puesto que en las primeras décadas persistía el dominio de las compañías especializadas. Expondremos cómo en los dos ramos tradicionales, incendios y transporte, nos encontramos con una elevada competencia en las primeras décadas que será menor en los otros tres ramos importantes debido a distintos motivos que explicaremos más adelante. El estudio por ramos también demostrará la fuerte presencia de las compañías extranjeras, lo que explicará determinadas estrategias de imitación de los mercados de origen. Sin embargo, a partir de la Primera Guerra Mundial el nacionalismo económico impregnará la política aseguradora gubernamental favoreciendo a las compañías nacionales, lo que provocará una progresiva «nacionalización» del negocio. A continuación se abordan dos aspectos nuevos en el estudio del seguro español. En el segundo apartado se analiza cómo el crecimiento de la competencia conducirá a las compañías que operan en el mercado español a estrategias individuales, como la diversificación, mediante la cual las compañías de seguro especializadas se convierten en compañías de seguro generales. Finalmente, en el tercer epígrafe veremos que, de forma paralela, en los ramos más competitivos se reforzará la cartelización, creando sindicatos y asociaciones para el control de la competencia. 


\section{EL CRECIMIENTO DE LA COMPETENCIA}

Las primas cobradas por las compañías de seguro en el mercado español crecieron entre 1912 y 1940 de forma constante a excepción de dos periodos '. En los cinco principales ramos (vida, incendios, accidentes de trabajo, accidentes individuales y responsabilidad civil y transportes) las primas (gráfico 1 y cuadro 1) explicitan este crecimiento, a excepción de los años 1916-1919 y de la Guerra Civil española. En estos dos períodos se produjo un fuerte descenso en la contratación de seguros. A pesar de que los datos que disponemos para 1937 y 1938 son parciales, puesto que sólo recogen las primas de la España Nacional, el impacto sobre el negocio del seguro es claro. Y aunque el indice de primas totales en 1940 recuperó los niveles de 1928, hay ramos en los que esta recuperación no se produjo hasta más tarde, como en el de incendios o accidentes individuales.

$\mathrm{El}$ análisis por ramos evidencia un desigual desarrollo. Crecieron incendios, vida, accidentes (de trabajo e individual y de responsabilidad civil) y transportes mientras se estancaron los ramos menores, tales como las tontinas, chatelusianas, ganado o enfermedad y defunciones ${ }^{2}$. Los ramos de vida e incendios siguieron creciendo y representando en todo el período en torno al 50 por 100 de las primas cobradas cada año, pero se percibe un descenso en el porcentaje que representaban respecto al total (cuadro 2). Si en 1912 ambos ramos suponían el 65 por 100 del total de las primas, este porcentaje descendió progresivamente hasta llegar a representar el 49,42 por 100 en 1935. Este descenso fue debido a un proceso de diversificación con el aumento de peso específico del ramo de accidentes de trabajo y de accidentes individuales y de responsabilidad civil en el negocio asegurador. El ramo de accidentes de trabajo creció durante la etapa de voluntariedad subsidiada que se inició en 1900, recibió un impulso con la reforma de la Ley en 1922, pero fue en 1933 cuando se produjo un

${ }^{1}$ Las primas oficiales sólo contabilizan las cantidades cobradas por las entidades aseguradoras inscritas en el registro especial que establece la Ley de 14 de febrero de 1908. Por tanto, quedan excluidas las primas negociadas por las entidades exceptuadas, muy numerosas en los ramos de enfermedad, entierros, ganados, cristales, accidentes de trabajo, etc. La mayor parte procedían de las sociedades de socorros mutuos creadas en el último tercio del siglo XIX (Castillo, 1994). El peso del mutualismo en el seguro francés en Gibaud (1997).

Un análisis pormenorizado de su evolución en Pons (2002a). Son ramos escasamente estudiados aunque se puede citar para las tontinas en el siglo XIX a Bahamonde (1981), para los seguros agrarios Burgaz y Pérez Morales (1996) y para enfermedad Rodriguez Ocaña (1990) y González Bueno (1986). En algunos casos su estancamiento se debe al desarrollo de los seguros sociales tales como el retiro obrero obligatorio desde 1919. Véase Cuesta (1988), Montero y Martínez (1988), Samaniego (1988) y Sudrià (1998). 


\section{GRÁFICO 1}

Evolución de las primas cobradas en los cinco principales ramos por las compañias de seguros en el mercado español (1912-1940). En pesetas constantes de 1928

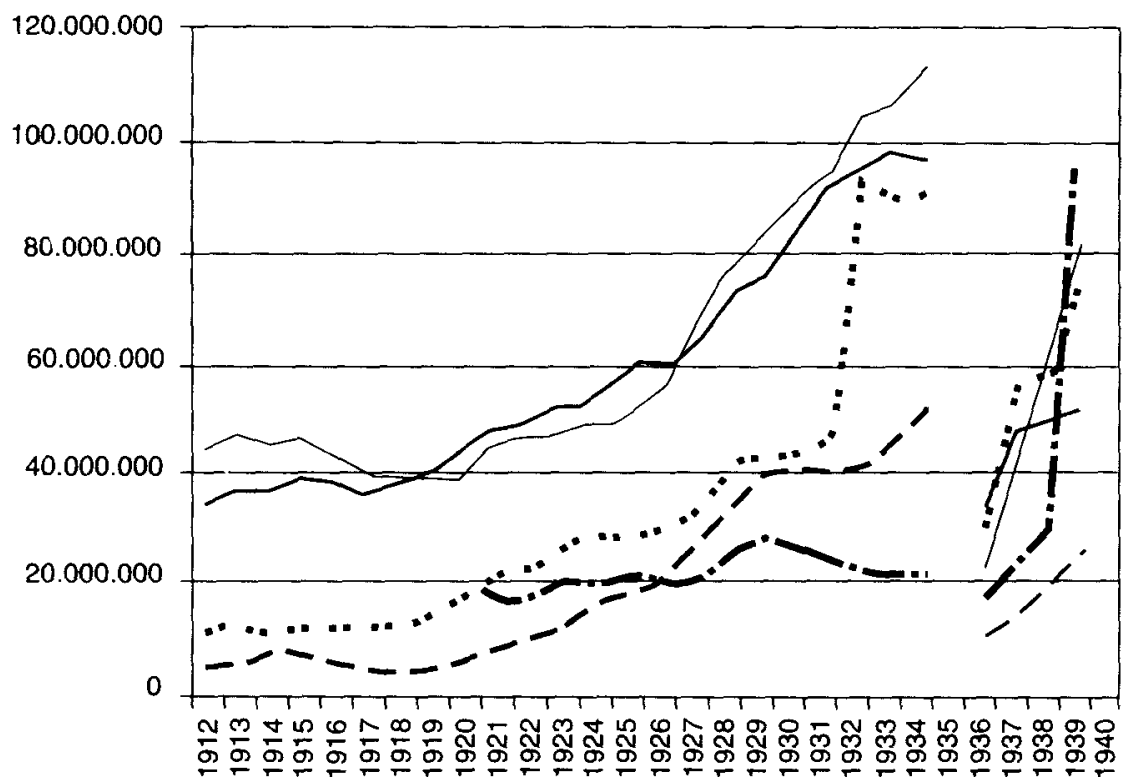

Vida $\ldots$ Incendios ... Accidentes de trabajo $\ldots$ - Accidentes individuales

. Transporte

FuENTE: Boletín Oficial de Seguros (1913-1926); Boletín Oficial de la Inspección Mercantil y de Seguros (1927-1928); Revista de Previsión (1929-1934); Boletín Oficial de Seguros y Ahorro (1934-1941); Anuario Estadístico de España (1919). En 1937 sólo se contemplan las primas en la España Nacional.

incremento espectacular con la puesta en práctica del seguro obligatorio aprobado por el gobierno republicano (Bibiloni y Pons, 1999). El seguro de accidentes de trabajo representaba sólo el 8 por 100 de las primas en 1912. El progreso fue lento pero significativo hasta 1920, en el que se situó en el 11,37 por 100 de las primas de seguros. Entre 1922, año de aprobación de la nueva Ley, y 1932 el porcentaje permaneció estable en torno al 13 por 100 del total cobrado por las compañías de seguro. Pero en 1933 la obligatoriedad del seguro duplicó el porcentaje representando el 22,67 por 100 de las primas cobradas en todos los ramos. 


\section{CUADRO 1}

Indice de las primas totales cobradas por las sociedades aseguradoras en España $y$ de los cinco principales ramos entre 1912-1940 (1928 = 100)

\begin{tabular}{|c|c|c|c|c|c|c|}
\hline Año & Vida & Incendio & $\begin{array}{c}\text { Accidentes } \\
\text { trabajo }\end{array}$ & $\begin{array}{c}\text { Accidentes } \\
\text { individ. }\end{array}$ & Transporte & Total \\
\hline 1912 & 62,0 & 50,4 & 27,8 & 11,7 & - & 44,4 \\
\hline 1913 & 65,3 & 54,7 & 32,5 & 13,8 & - & 49,2 \\
\hline 1914 & 63,4 & 54,0 & 28,8 & 18,1 & - & 49,6 \\
\hline 1915 & 65,4 & 58,3 & 31,9 & 15,9 & - & 52,7 \\
\hline 1916 & 61,6 & 57,1 & 33,3 & 15,2 & - & 51,8 \\
\hline 1917 & 56,5 & 54,0 & 33,7 & 14,4 & - & 49,3 \\
\hline 1918 & 55,2 & 56,3 & 35,5 & 13,7 & - & 49,0 \\
\hline 1919 & 55,1 & 59,5 & 36,6 & 14,6 & - & 49,3 \\
\hline 1920 & 55,1 & 64,4 & 45,8 & 18,0 & - & 51,6 \\
\hline 1921 & 62,6 & 72,0 & 56,0 & 25,8 & 89,8 & 66,3 \\
\hline 1922 & 66,1 & 73,7 & 67,1 & 30,6 & 78,5 & 68,5 \\
\hline 1923 & 66,2 & 78,2 & 70,4 & 36,1 & 82,3 & 70,7 \\
\hline 1924 & 69,4 & 80,3 & 80,9 & 45,4 & 96,0 & 76,4 \\
\hline 1925 & 69,9 & 85,6 & 84,6 & 61,1 & 94,3 & 80,2 \\
\hline 1926 & 76,6 & 92,8 & 83,1 & 67,4 & 102,7 & 86,2 \\
\hline 1927 & 82,2 & 92,9 & 90,7 & 77,6 & 95,2 & 89,0 \\
\hline 1928 & 100,0 & 100,0 & 100,0 & 100,0 & 100,0 & 100,0 \\
\hline 1929 & 112,9 & 112,9 & 124,9 & 125,5 & 125,0 & 115,6 \\
\hline 1930 & 121,3 & 117,1 & 125,6 & 144,4 & 135,3 & 123,6 \\
\hline 1931 & 130,0 & 128,7 & 130,2 & 147,2 & 124,5 & 128,5 \\
\hline 1932 & 136,4 & 142,5 & 139,5 & 147,2 & 116,4 & 134,3 \\
\hline 1933 & 151,5 & 146,6 & 279,1 & 153,0 & 107,2 & 157,7 \\
\hline 1934 & 154,3 & 151,7 & 272,4 & 164,4 & 104,5 & 159,7 \\
\hline 1935 & 163,8 & 149,4 & 269,4 & 189,7 & 106,6 & 163,5 \\
\hline 1936 & - & - & - & - & - & - \\
\hline 1937 & 31,2 & 49,6 & 78,0 & 40,3 & 82,6 & 43,1 \\
\hline 1938 & 59,0 & 73,8 & 169,8 & 53,3 & 118,4 & 80,6 \\
\hline 1939 & 86,6 & 76,9 & 175,2 & 74,9 & 148,3 & 92,0 \\
\hline 1940 & 118,0 & 80,1 & 227,1 & 94,0 & 466,3 & 136,1 \\
\hline
\end{tabular}

FUENTE: Boletin Oficial de Seguros (1913-1926); Boletín Oficial de la Inspección Mercantil $y$ de Seguros (1927-1928); Revista de Previsión (1929-1934); Boletín Oficial de Seguros y Aborro (1934-1941); Anuario Estadístico de España (1919). Conocemos datos para 1936 pero son muy parciales. En 1937 sólo se contemplan las primas en la España Nacional. 


\section{CUADRO 2}

Porcentaje de las primas por ramos sobre el total de las primas recaudadas, 1912-1940

\begin{tabular}{|c|c|c|c|c|c|c|c|}
\hline$A \bar{n} o$ & Vida & Incendios & $\begin{array}{c}\text { Accidentes } \\
\text { trabajo }\end{array}$ & $\begin{array}{l}\text { Accidentes } \\
\text { individ. }\end{array}$ & Transporte & $\begin{array}{l}\text { Otros } \\
\text { ramos }\end{array}$ & Total \\
\hline 1912 & 37,17 & 28,28 & 8,01 & 2,77 & - & 23,77 & 100 \\
\hline 1913 & 35,30 & 27,66 & 8,45 & 2,94 & - & 25,65 & 100 \\
\hline 1914 & 33,98 & 27,11 & 7,45 & 3,84 & - & 27,62 & 100 \\
\hline 1915 & 33,04 & 27,54 & 7,74 & 3,17 & - & 28,51 & 100 \\
\hline 1916 & 31,62 & 27,46 & 8,23 & 3,08 & - & 29,61 & 100 \\
\hline 1917 & 30,52 & 27,31 & 8,75 & 3,06 & - & 30,36 & 100 \\
\hline 1918 & 29,99 & 28,63 & 9,28 & 2,93 & - & 29,17 & 100 \\
\hline 1919 & 29,73 & 30,06 & 9,51 & 3,11 & - & 27,59 & 100 \\
\hline 1920 & 28,45 & 31,11 & 11,37 & 3,67 & - & 25,40 & 100 \\
\hline 1921 & 25,12 & 27,03 & 10,81 & 4,09 & 10,66 & 22,29 & 100 \\
\hline 1922 & 25,70 & 26,81 & 12,54 & 4,69 & 9,02 & 21,24 & 100 \\
\hline 1923 & 24,92 & 27,54 & 12,75 & 5,36 & 9,16 & 20,27 & 100 \\
\hline 1924 & 24,19 & 26,20 & 13,56 & 6,25 & 9,89 & 19,91 & 100 \\
\hline 1925 & 23,21 & 26,59 & 13,51 & 8,00 & 9,26 & 19,43 & 100 \\
\hline 1926 & 23,66 & 26,83 & 12,34 & 8,22 & 9,38 & 19,57 & 100 \\
\hline 1927 & 24,57 & 25,99 & 13,05 & 9,16 & 8,42 & 18,81 & 100 \\
\hline 1928 & 26,62 & 24,91 & 12,80 & 10,50 & 7,87 & 17,30 & 100 \\
\hline 1929 & 25,99 & 24,33 & 13,83 & 11,40 & 8,51 & 15,94 & 100 \\
\hline 1930 & 26,13 & 23,60 & 13,01 & 12,27 & 8,62 & 16,37 & 100 \\
\hline 1931 & 26,94 & 24,95 & 12,97 & 12,04 & 7,63 & 15,47 & 100 \\
\hline 1932 & 27,04 & 26,44 & 13,29 & 11,51 & 6,82 & 14,90 & 100 \\
\hline 1933 & 25,58 & 23,16 & 22,67 & 10,19 & 5,35 & 13,05 & 100 \\
\hline 1934 & 25,72 & 23,67 & 21,84 & 10,81 & 5,15 & 12,81 & 100 \\
\hline 1935 & 26,66 & 22,76 & 21,09 & 12,18 & 5,13 & 12,18 & 100 \\
\hline 1936 & - & - & - & - & - & - & - \\
\hline 1937 & 19,28 & 28,67 & 23,19 & 9,84 & 15,10 & 3,92 & 100 \\
\hline 1938 & 19,50 & 22,83 & 26,98 & 6,94 & 11,57 & 12,18 & 100 \\
\hline 1939 & 25,05 & 20,81 & 24,37 & 8,56 & 12,69 & 8,52 & 100 \\
\hline 1940 & 23,07 & 14,66 & 21,35 & 7,25 & 26,97 & 6,7 & 100 \\
\hline
\end{tabular}

FUENTE: Boletin Oficial de Seguros (1913-1926); Boletin Oficial de la Inspección Mercantil $y$ de Seguros (1927-1928); Revista de Previsión (1929-1934); Boletín Oficial de Seguros y Aborro (1934-1941); Anuario Estadistico de España (1919). Conocemos datos para 1936 pero son muy parciales. En 1937 sólo se contemplan las primas en la España Nacional. 
Por su parte, los accidentes individuales y de responsabilidad civil pasaron del 2,77 por 100 en 1912 al 12,18 por 100 en 1935.

El ramo de transporte tuvo grandes oscilaciones vinculadas al aumento de las primas en período de guerra. Hasta 1920 las compañías de seguro que operaban en el ramo estaban exceptuadas y se regian por el Código de Comercio. El Real Decreto de 13 de agosto de 1920 obligó a las empresas que operaban en el seguro de transportes a solicitar la inscripción en el registro oficial establecido por la Ley de 14 de mayo de 1908. Esta obligación supuso un cambio esencial en el negocio por los nuevos requisitos establecidos por la Administración (depósitos, reservas, declaración de primas, etc.). Muchas compañias extranjeras optaron por la liquidación de sus sucursales en España y también se disolvieron algunas españolas. En cuanto al volumen de primas el comportamiento del ramo fue muy irregular. En los primeros años veinte este negocio presentaba fuertes oscilaciones que en la segunda mitad de la década parecían superadas con un crecimiento progresivo de las primas hasta 1930, año en que se inauguró una inflexión provocada por la depresión mundial y la reducción del comercio en general. La Guerra Civil española y el estallido del conflicto mundial con el incremento de las primas de guerra dispararon el volumen de las primas del ramo, triplicándolas en 1940 con respecto a 1939. Las primas de transporte que representaban un 10,66 por 100 de las primas de seguros en 1921 sufrieron una caída hasta representar sólo un 5,13 por 100 en 1935, pero durante la Guerra Civil representaron de nuevo más del 10 por 100 para situarse en el 27 por 100 en 1940.

Uno de los aspectos más significativos en los ramos en los que se produjo un crecimiento importante del volumen de primas es el aumento del número de compañías autorizadas para operar en cada ramo. De las 138 inscritas en 1911 se había pasado a 263 en 1921, 364 en 1931 y 397 en $1940^{3}$. La autorización tan elevada de compañías provocó un crecimiento de la competencia en muchos ramos con la consiguiente guerra de tarifas, aunque en algunos aseguraban compañías que tenían un nivel tan bajo de recaudaciones que difícilmente cubrian los gastos de explotación ${ }^{4}$.

${ }^{3}$ El número de compañías creció a pesar de las restricciones oficiales como la elevación de los depósitos en 1927 o la suspensión de las inscripciones desde 1949 hasta 1952. La Estrella (1959, p. 107).

4 Esta característica persistió en el mercado español incluso en los años cincuenta. En 1957 España tenía 668 compañías autorizadas frente a las 135 de Italia, 86 de Suiza, 412 de Inglaterra y sólo era superada por Francia con 686. Sin embargo, de las compañías 
El crecimiento de la competencia se presentó sobre todo en dos ramos: incendios y transportes. Entre los factores explicativos habría que resaltar que fueron los dos ramos de mayor desarrollo en el siglo Xx. En ambos operaban una elevada proporción de compañías extranjeras con una larga presencia en nuestro mercado. En el caso del ramo de incendios a las compañías de ámbito nacional y a las foráneas cabe añadir pequeñas mutualidades y compañías mercantiles que se habían fundado en casi todas las capitales de provincia en las últimas décadas del siglo XIX. Sin embargo, la mayor dificultad técnica y la exigencia de mayores reservas impidieron durante las dos primeras décadas de siglo el crecimiento del número de compañías en el ramo de vida. Por su parte, los ramos de accidentes, de trabajo e individuales o de responsabilidad civil, empezaban a desarrollarse a principios de siglo.

Es precisamente en el ramo de incendios donde se había manifestado una fuerte competencia en el mercado inglés durante el siglo $\mathrm{XIX}^{5}$. En España, si relacionamos el aumento del número de compañías que operaban en este ramo con el incremento de las primas se observará un escaso crecimiento de las primas por sociedad. En 1912 un total de 46 entidades aseguraban por valor de 32.926 .139 pesetas, con lo que la media de primas por compañía se situaba en 715.785 pesetas. En los años sucesivos se incrementó el número de sociedades y, sin embargo, la media por empresa sufrió un escaso aumento. En 1919 las 45 aseguradoras que operaban obtuvieron una media de 863.238 pesetas en concepto de primas mientras que la media de las 59 compañías de 1924 se situó en 888.501 pesetas. El progresivo incremento de nuevas empresas en este ramo dificultó el crecimiento del negocio. Desde 1912 a 1926 la media de primas por sociedad no superó las 900.000 pesetas con pequeñas oscilaciones, a excepción de 1922. En 1927 las 66 aseguradoras activas en este seguro obtuvieron unas primas medias por valor de 918.331 pesetas y al año siguiente el nuevo incremento de entidades hasta 71 mantuvo la media en 918.745 pesetas. La estabilidad en el número de empresas que negociaban con este tipo de seguro -alrededor de 71 entre 1928 y 1932 - permitió cierto incremento de la media hasta llegar a 1.300 .000 pesetas. De nuevo se produjo un descenso en la media en los tres años siguientes cuando el número

españolas autorizadas sólo 92 cobraban primas por valor superior a los 15 millones de pesetas. La Estrella (1959, p. 105).

'Sobre los efectos de la competencia en el mercado inglés, sobre todo en el ramo de incendios, véase Jenkins (1984), Ryan (1984), Supple (1984), Pearson (1991 y 1993), Cockerell y Grenn (1994, pp. 26-56) y Westall (1984, 1997 y 1998). 
de las compañías que contratan el seguro de incendios llegó a 80. A estos datos habría que añadir informaciones cualitativas que aparecieron en la prensa especializada de la época que hacian referencia a la competencia existente en el ramo de incendios y en el de transportes ${ }^{6}$.

Sin embargo, las exigencias técnicas y de capital de las empresas de vida, durante las dos primeras décadas del siglo XX, se tradujeron en un menor incremento de la competencia en el ramo de vida. Este ramo presentaba un marcado carácter oligopólico. Las mismas 23 compañías que aseguraban en el mercado en 1912 se mantuvieron hasta 1925 (oscilaron entre 23 y 25). Sin embargo, el estancamiento de las primas en este periodo mantuvo las primas medias por compañía bastante estable en torno a los 2 millones de pesetas. Desde 1926 hasta 1935 se sumaron lentamente nuevas compañías hasta alcanzar las 43 . El crecimiento progresivo de las primas en esta nueva etapa de los cerca de 53 millones a los 114 de 1935, hizo que la media de primas por compañía se elevara de 2 a 2,5 millones de pesetas.

En los ramos nuevos el incremento de la competencia se retrasó hasta la década de los años treinta. En el ramo de accidentes de trabajo ${ }^{7}$, creado tras la Ley de Accidentes de Trabajo de 1900, sólo 15 compañias operaban en 1912, cifra que no se duplicó hasta 1928. Un año después se consiguió doblar la media de primas por compañía con respecto a 1912. El crecimiento espectacular se produjo con la obligatoriedad del seguro en 1933 cuando de nuevo se duplica la media de primas por entidad. Este resultado se obtuvo con la incorporación de sólo nueve compañías. Sin embargo, en este ramo se incrementó en gran medida la competencia, no por la incorporación de compañías mercantiles, sino por la proliferación de mutualidades patronales de accidentes de trabajo y la creación de la Caja Nacional de seguro de accidentes de trabajo en 1932 (Pons, 1998, y Bibiloni y Pons, 1999).

Junto al fenómeno descrito del crecimiento de la competencia derivado del aumento del número de compañías por ramo cabe hablar del proceso de la «nacionalización del mercado». Este cambio fue debido no sólo al abandono de sociedades extranjeras en los ramos principales, sino por la

\footnotetext{
${ }^{6}$ A modo de ejemplo véase para el seguro de incendios un artículo sobre la «Asamblea de Compañias de seguros contra incendios», en Los Seguros, p. 23 del 15 de marzo de 1918 , y para el caso de transportes «Las compañías españolas de seguros», en El eco del seguro, p. 6 de abril de 1926 . Otras referencias en el epigrafe $3^{\circ}$ donde se analiza la cartelización.

'El desarrollo de este seguro fue distinto al inglés (Dinsdale, 1954) y norteamericano (Fishback y Kantor, 1996 y 1998).
} 
incorporación masiva de nuevas empresas nacionales. Si analizamos el número total de aseguradoras que operaban en España, según las estadísticas oficiales y en términos absolutos, se puede comprobar que desde 1911 hasta 1940 las compañías nacionales representaban en torno al 60 por 100 del total. Entre 1911 y 1919 las compañías extranjeras suponían aproximadamente el 35 por 100 , porcentaje que experimentó un incremento en la década siguiente debido a la incorporación del ramo de transportes al registro general de entidades de seguros implantado tras la Ley del 14 de febrero de 1908. Hasta 1932 el porcentaje de participación de entidades foráneas se situará alrededor del 40 por 100 , siendo 1926 , con el 43 por 100, el año de mayor participación. A partir de 1929 el peso de la participación extranjera disminuyó, descendiendo al 35 por 100 en 1934. El mercado de seguros de la posguerra confirmó esta tendencia situándose en el 24 por 100 en 1944, el porcentaje más bajo de todo el período ${ }^{8}$. Las primas cobradas por las compañías extranjeras que ofrecen Frax y Matilla (1996) corroboran su peso en el mercado entre 1912 y 1934. En el primer año cobraban el 40 por 100 de las primas y, aunque hay un leve descenso en los siguientes años, aún en 1934 ingresaban el 34 por 100 de las primas.

Sin embargo, los datos globales del número de compañías que operan en España no reflejan la realidad, puesto que en los ramos donde el negocio es menor, tales como el de enfermedades y defunciones o las tontinas y chatelusianas, prácticamente sólo participaban entidades espanolas. Si analizamos el porcentaje que representan las compañías extranjeras en los principales ramos (vida, incendios, accidentes de trabajo y accidentes individuales, responsabilidad civil y transporte) el panorama es otro (cuadro 3 ). En 1912 las compañías extranjeras representaban el 65 por 100 de las entidades en el seguro de vida, el 54 por 100 en el de incendio ${ }^{9}$ y el 63 por 100 en el de accidentes individuales. En 1921, primer año del que disponemos estadísticas, las compañías extranjeras suponían el 61 por 100 del total de entidades en el seguro de transportes. En el único ramo en el que en 1912 las entidades aseguradoras extranjeras no eran mayoría

${ }^{*}$ El crecimiento de las compañías nacionales se acelera posteriormente durante la Segunda Guerra Mundial y en 1954 con la inscripción gratuita en nuevos ramos. La Estrella (1959, p. 102).

' Las compañias extranjeras más numerosas en este ramo eran las inglesas. La fuerte competencia el mercado inglés a mediados del siglo XIX (Westall, 1994) impulsó lá creación en 1869, del Fire Offices' Committee (Foreign), formado por 29 compañías, para establecer acuerdos sobre tarifas en el extranjero. Cockerell y Green (1994, p. 40). 


\section{CUADRO 3}

Número de compañias por nacionalidad de los principales ramos en el mercado del seguro español (1912-1940)

\begin{tabular}{|c|c|c|c|c|c|c|c|c|c|c|c|c|c|c|c|}
\hline \multirow[t]{2}{*}{ Año } & \multicolumn{3}{|c|}{ Vida } & \multicolumn{3}{|c|}{ Incendios } & \multicolumn{3}{|c|}{$\begin{array}{l}\text { Accidentes } \\
\text { de trabajo }\end{array}$} & \multicolumn{3}{|c|}{$\begin{array}{c}\text { Accidentes } \\
\text { individuales y } \\
\text { responsabilidad } \\
\text { civil }\end{array}$} & \multicolumn{3}{|c|}{ Transportes } \\
\hline & h & 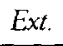 & & Esp. & $F_{x}$ & & & $F_{x}$ & Total & & Ext. & Total & & Ext. & \\
\hline 1912 & 8 & 15 & 23 & 17 & 25 & 46 & 9 & 6 & 1 & 4 & 7 & 11 & - & - & \\
\hline 1913 & 8 & 15 & 23 & 17 & 30 & 47 & 12 & 8 & 20 & 4 & 9 & 13 & - & 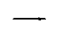 & \\
\hline 1914 & 8 & 14 & 22 & 17 & 30 & 47 & 13 & 9 & 22 & 5 & 11 & 16 & - & $\ldots$ & - \\
\hline 1915 & 8 & 14 & 22 & 17 & 30 & 47 & 13 & 8 & 21 & 5 & 11 & 16 & - & $\ldots$ & - \\
\hline 1916 & 8 & 14 & 22 & 16 & 31 & 47 & 12 & 9 & 21 & 5 & 12 & 17 & - & - & - \\
\hline 1917 & 9 & 14 & 23 & 13 & 30 & 43 & 14 & 9 & 23 & 6 & 12 & 18 & - & - & $\ldots$ \\
\hline 1918 & 9 & 16 & 2) & 17 & 31 & 40 & 19 & 7 & 26 & 7 & 12 & 19 & - & $\ldots$ & \\
\hline 1919 & 9 & 15 & 2 & 15 & 30 & 45 & 14 & 9 & 23 & 6 & & 19 & - & - & \\
\hline 192 & 8 & 15 & 23 & 21 & 31 & 5 & 14 & 0 & 24 & 8 & 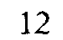 & 20 & - & - & - \\
\hline 1921 & 8 & 15 & 23 & 17 & 35 & 52 & 13 & 11 & 24 & 8 & 14 & 22 & 16 & 26 & 42 \\
\hline 1922 & 9 & 14 & 23 & 18 & 33 & 51 & 12 & 11 & 23 & 7 & 16 & 23 & 17 & 33 & 50 \\
\hline 1923 & 10 & 14 & 24 & 23 & 34 & 57 & 15 & 13 & 28 & 9 & 16 & 25 & 21 & 32 & 53 \\
\hline 1924 & 11 & 14 & 25 & 24 & 35 & 59 & 15 & 15 & 30 & 10 & 16 & 26 & 18 & 34 & 52 \\
\hline 1925 & 11 & 14 & 26 & 30 & 36 & 66 & 15 & 14 & 29 & 13 & 17 & 30 & 17 & 35 & 52 \\
\hline 1926 & 12 & 14 & 26 & 32 & 35 & 71 & 14 & 15 & 29 & 13 & 17 & 35 & 17 & 34 & 51 \\
\hline 1927 & 12 & 14 & 27 & 29 & 37 & 66 & 11 & 16 & 27 & 11 & 20 & 31 & 27 & 22 & 49 \\
\hline 1928 & 12 & 1 & 29 & 3 & 39 & 71 & 13 & 17 & 30 & 14 & 19 & & 18 & 1 & 49 \\
\hline 1925 & 1. & 1 & 29 & 29 & 42 & 71 & 17 & 15 & 32 & 16 & 20 & 36 & 26 & 3 & 49 \\
\hline 1930 & 1 & 15 & 30 & 31 & 43 & 74 & 19 & 15 & 34 & 20 & 20 & 0 & 16 & 4 & 50 \\
\hline 1931 & 17 & 16 & 33 & 31 & 43 & 74 & 20 & 16 & 36 & 19 & 21 & 40 & 20 & 28 & 48 \\
\hline 1932 & 19 & 18 & 37 & 31 & 40 & 71 & 19 & 16 & 35 & 19 & 19 & 38 & 18 & 25 & 43 \\
\hline 1933 & 21 & 18 & 39 & 32 & 42 & 74 & 21 & 16 & 37 & 21 & 20 & 41 & 19 & 25 & 44 \\
\hline 1934 & 24 & 15 & 43 & 34 & 45 & 79 & 22 & 16 & 38 & 20 & 23 & 43 & 19 & 26 & 45 \\
\hline 1935 & 27 & 16 & 43 & 37 & 43 & 80 & 23 & 16 & 39 & 19 & 23 & 42 & 20 & 26 & 46 \\
\hline 1936 & - & - & - & - & - & - & - & - & - & - & - & - & - & - & - \\
\hline 1937 & 24 & 16 & 40 & 31 & 40 & 71 & 21 & 15 & 36 & 20 & 21 & 41 & 15 & 21 & 36 \\
\hline 1938 & 26 & 18 & 44 & 30 & 40 & 70 & 19 & 15 & 34 & 19 & 20 & 39 & 21 & 25 & 46 \\
\hline 193 & 26 & 18 & 44 & 35 & 44 & 7 & 23 & 14 & 37 & 22 & 20 & 42 & 21 & 25 & 46 \\
\hline 1940 & 26 & 18 & 44 & 40 & 43 & 83 & 28 & 16 & 44 & 25 & 20 & 45 & 22 & 25 & 47 \\
\hline
\end{tabular}

FuenTE: Boletin Oficial de Seguros (1913-1926); Boletin Oficial de la Inspección Mercantil $y$ de Seguros (1927-1928); Revista de Previsión (1929-1934); Boletín Oficial de Seguros y Aborro (1934-1941); Anuario Estadístico de España (1919). Conocemos datos para 1936 pero son muy parciales. En 1937 sólo se contemplan las primas en la España Nacional. 
era en el de accidentes de trabajo en el que representaban sólo el 40 por 100. Esta tónica persistió a lo largo de todo el período estudiado.

En el ramo de vida las compañías nacionales no llegaron a alcanzar el 50 por 100 del total hasta 1930 , en el de incendios nunca lo superaron, en el ramo de transportes sólo sobrepasaron a las extranjeras en 1929 y en el de accidentes individuales sólo lo conseguieron tras la Guerra Civil. Por tanto, las compañías extranjeras dominaron el mercado asegurador español, al ser mayoritarias en los ramos que negociaban el volumen mayor de primas ${ }^{10}$. Las compañías extranjeras fueron dominantes durante las dos primeras décadas del siglo y tras la Primera Guerra Mundial hasta los años treinta, período en el que experimentaron un progresivo descenso, en parte debido al nacionalismo económico que inundó la política aseguradora de la mayor parte de gobiernos y entre cuyas consecuencias estuvo el abandono de algunos mercados por parte de importantes corporaciones multinacionales (Keller, 1963).

En España las primeras intervenciones respecto a la creación de limitaciones en la actuación de las compañías de seguros extranjeras aparececieron tras la Primera Guerra Mundial con el control sobre el máximo en la inversión de reservas en el extranjero, reforzadas más tarde con la Real Orden del 6 de abril de 1925 que prohibió que las empresas aseguradoras continuasen invirtiendo fuera de España el 50 por 100 de sus reservas matemáticas y hasta el 60 por 100 de las de riesgo en curso, hecho que perjudicaba directamente a las compañías extranjeras ${ }^{11}$. Éstas vieron atacados sus intereses y como reacción se asociaron creando la Agrupación Española de Compañias Extranjeras de Seguros que mantendría una gran actividad en esta década. En febrero de 1926 integraban la asociación trece compañías inglesas, diez francesas, una italiana, una suiza y una sueca del ramo de incendios. La mayor parte estaban instaladas en Madrid (13 entidades) y Barcelona (8). El resto se repartían entre Bilbao y Málaga con dos compañías por una en San Sebastián. También eran miembros doce compañías que operaban en el ramo de transportes - diez de ellas en Barcelona y dos en Bilbao-. Esta política en el campo asegurador continuó con un polémico Real Decreto de 18 de febrero de 1927, com-

10 El peso del capital extranjero en el sector sería aún mayor si se analizara el accionariado de algunas compañias que aparecen como nacionales y en realidad una parte impor. tante de su capital es extranjero. El caso más conocido es el de La Unión y el Fénix (Frax y Matilla, 1996, p. 196).

"Sobre la evolución de las inversiones de las compañias aseguradoras, Pons (2001) y Gallegos (1967). Para el ramo de vida en Francia véase Hautcoeur y Verley (1998). 
plementado con otro de 27 de noviembre del mismo año que establecía que las compañías extranjeras que deseaban operar en España debían efectuar depósitos complementarios cuando no hubiesen desembolsado el 25 por 100 del capital suscrito, a pesar de sus reservas estatutarias que no eran computables en este caso.

Esta normativa creó un gran malestar entre las compañías de seguro extranjeras (Pons, 2001). Los rumores sobre la nacionalización del seguro ${ }^{12}$ se extendieron en el sector, con el precedente de las limitaciones citadas con anterioridad, y tras el discurso del Ministro de Hacienda realizado el 20 de enero de 1928, provocando la publicación de una nota oficiosa procedente del Presidente del Consejo de Ministros en la que declaraba que jamás se había pensado en nada que significara irrespetuosidad para los derechos reconocidos en convenios internacionales. Este clima proteccionista influyó en que abandonaran el mercado español, sobre todo en el ramo de vida, veteranas compañías como las norteamericanas The New York Life Insurance Company, The Equitable Life Assurance Society of The United States (Pons, 2002b) o la inglesa Standard Life Assurance (Butt, 1984).

$\mathrm{El}$ incremento de la competencia y el peso de las empresas extranjeras en el mercado son dos de las claves que nos permiten comprender las estrategias que desarrollaron las compañías que operaban en España. Si bien algunas empresas internacionales se especializaron para aprovechar las economías de escala, como el caso de las mutualidades de vida norteamericanas, las compañias extranjeras y nacionales, por su parte, ante la incorporación de nuevas entidades optaron por la diversificación. Muchas sociedades en su origen especializadas en un solo ramo fueron ampliando sus actividades a otros tipos de seguros hasta convertirse en compañías de seguros generales.

\section{DE LAS COMPAÑÍAS ESPECIALIZADAS A LAS COMPAÑÍAS GENERALES DE SEGUROS}

Las compañías españolas y extranjeras creadas a lo largo del siglo XIX y que operaban en el mercado español a principios del siglo Xx se habían fundado, en la mayor parte de los casos, para participar en un solo ramo. Se trataba, por tanto, de compañías especializadas. De esta forma, El Banco Vitalicio de España, nacido de la fusión de La Previsión y el Banco Vitalicio

12 El caso de la nacionalización de los seguros de vida en Italia vease Fanfani (1998) o la municipalización de los seguros en Portugal analizada por Romero Magalhaes (1998). 
de Cataluña, operaba en las diversas modalidades del seguro de vida, mientras que la Previsión Española y la Sociedad Catalana de Seguros contra incendios operaban únicamente en el ramo de incendios. El crecimiento de la competencia, localizada sobre todo en los ramos tradicionales como el de incendios, impulsó la diversificación de las compañías empezando a participar en nuevos ramos para aprovechar de forma más intensiva sus instalaciones, recursos humanos y financieros. Comparativamente este fenómeno se desarrolló en Francia en la primera década del siglo Xx (Frax y Matilla, 1998) y en el caso de Gran Bretaña el mismo proceso se inició en la última década del siglo XIX (Cockerell y Green, 1994).

En España la diversificación no fue homogénea e incluso algunas compañias insistieron en la especialización. Una de las compañías líderes en el ramo de incendios, la Sociedad Catalana de Seguros contra Incendios creada en 1864, optó por las ventajas derivadas del tamaño del mercado adquiriendo carteras de otras compañías y en todo el período comprendido entre 1912 y 1940 sólo operó en este ramo. En 1916 adquirió la cartera de incendios de Hispania y en 1921 la cartera de Celtiberia. Esta sociedad mantuvo su política de especialización hasta 1947, año en que claudicó ante el evidente éxito de la diversificación del resto de compañías y empezó a operar en los ramos de vida, accidentes individuales y colectivos y daños a vehículos (Catalana Occidente, 1989 y Pons, 2000). Sin embargo esta política se complementó con la creación de filiales o la participación en otras empresas especializadas en otros ramos del seguro o en el de incendios. Es el caso de su intervención en la Previsión Nacional, la compañía Covadonga, el Banco Vitalicio de España o la Caja de Previsión y Socorro. $\mathrm{Al}$ menos en el consejo de administración de todas estas sociedades encontramos integrantes de la dirección de La Catalana ${ }^{13}$.

También prefirió la especialización la compañía Hispania que abandonó «incendios» en 1916 para concentrar sus esfuerzos en los ramos menos competitivos de accidentes de trabajo, accidentes individuales y de responsabilidad civil. Pero se trata de casos excepcionales entre las compañías españolas, a las que habría que añadir las compañías extranjeras especializadas sobre todo en el ramo de vida que, debido a los mayores requisitos

${ }^{13}$ En la primera junta de gobierno del Banco Vitalicio de España de 1898 se encuentran entre los vocales el Marqués de Alella, Fernando Delás y José María Delás, que son directivos destacados de la Sociedad Catalana de Seguros conira incendios. Banco Vitalicio de España (1980), p. 14, y Catalana Occidente (1989), p. 29. El conocimiento de los accionistas de ambas entidades seguramente nos daría más información sobre la relación entre la propiedad de las dos entidades. 
técnicos y actuariales, actuaban en un mercado de carácter oligopólico. De esta forma en el seguro de vida operan compañías multinacionales que durante todo el período estudiado no presentan ningún interés en participar en otros ramos del mercado español. Es el caso de las norteamericanas The New York Life Insurance Company, The Equitable Life Assurance Society of The United States o The Guardian Life Insurance Company of America o la brasileña Equitativa dos Estados Unidos do Brazil. También mantendrán la especialización algunas compañias extranjeras inscritas en el ramo de transportes, como la italiana Italia y las alemanas Mannbeim, Dusseldorf, Hansa de Hamburgo, Badense y Francfort.

Sin embargo, la extensión de sus actividades a otros ramos se convertirá en un fenómeno generalizado entre la mayor parte de compañias nacionales y extranjeras. La diversificación afecta exclusivamente a los ramos de vida, incendios, transportes, accidentes de trabajo y accidentes individuales o de responsabilidad civil. La nueva Ley en el seguro de transportes y la reforma de la Ley de Accidentes de Trabajo en 1920 y 1922, respectivamente, impulsaron este proceso. El resto de ramos - enfermedad y defunciones, ganados, cristales, pedrisco o tontinas-- permaneció en manos de pequeñas compañias especializadas hasta la Guerra Civil.

En el proceso de diversificación se dan tres etapas ${ }^{14}$. La primera etapa, de exploración, abarca de 1900 a 1920 cuando unas pocas compañías españolas y extranjeras iniciaron la ampliación de sus operaciones originales hacia otros ramos. Entre las compañías españolas precursoras estarian la Caja de Previsión y Socorro, que aseguraba en 1912 en el ramo de vida, accidentes de trabajo y accidentes individuales, o La Estrella, que también negociaba en 1912 con seguros de vida, incendios y accidentes de trabajo y que en 1914 amplió su actividad a los accidentes individuales y, en 1923, a los transportes. Entre las más precoces habría que incluir a La Unión y el Fénix Español, que ya en el siglo $\mathrm{xIX}$ se puede considerar compañía de seguros generales. En 1912 participaba en el ramo de vida, incendios y transporte, en 1917 amplió sus operaciones a los accidentes individuales y en 1918 a los accidentes de trabajo. Entre las compañías extranjeras de diversificación temprana encontramos a la francesa L'Abeille, ya consolidada como compañía de seguros generales en su país y que desde 1912 estaba presente en los ramos de vida, incendios y accidentes de trabajo $\mathrm{e}$ individuales.

${ }^{14}$ La relación completa de las compañías que operan en los seis ramos principales y su proceso de diversificación se encuentra en Pons (2002a). 
En la década de 1920 se da una segunda etapa, en la que el anterior proceso de exploración se intensifica hasta consolidarse. Entre las compañías españolas más destacadas que se diversifican durante esta etapa se encuentra el Banco Vitalicio de España, compañía especializada desde su nacimiento en las diferentes modalidades del ramo de vida y que en 1921, aprovechando la reestructuración del ramo, inició sus operaciones en el de transportes y en 1925, en el de incendios. Un segundo ejemplo lo ofrece el Centro Catalán de Aseguradores, que, especializado en el seguro marítimo, haría coincidir su transformación en Plus Ultra en 1924 con la ampliación de sus actividades a los ramos de vida, incendios y accidentes individuales, y al que añadiría a partir de 1931 los accidentes de trabajo. Las compañías extranjeras, por su parte, también participaron de este proceso. La francesa L'Union, especializada en el ramo de incendios y con sede en Barcelona, ofrecía desde 1919 seguros de vida y, desde 1922, accidentes de trabajo e individuales, pero ya domiciliados en su sede de Madrid. No es éste un hecho aislado, ya que muchas compañías francesas presentan un doble domicilio social según el ramo, lo que determina cierta especialización entre Madrid y Barcelona, característica ya destacada por Frax y Matilla (1996) para las primeras décadas de siglo xx. Es el caso de La Preservatrice, constituida en París en 1874 como continuadora de una compañía del mismo nombre que tenía por objeto principal el seguro de accidentes. Se estableció en Barcelona en 1908 inscribiéndose en el ramo de accidentes de trabajo y accidentes individuales. Cuando en 1928 empezó a asegurar en transportes señaló como domicilio social su sucursal de Madrid (Tortella, 2000). De igual forma, L'Abeille mantuvo el domicilio social en el ramo de seguro de incendios en Madrid y el ramo de vida y los ramos de accidentes de trabajo e individuales en Barcelona ${ }^{15}$. Las compañias inglesas especializadas en el competitivo ramo de incendios que operaron en el mercado español también ramifican sus actividades, aunque generalmente este proceso de diversificación ya lo había iniciado su casa matriz. La London \& Lancashire ${ }^{16}$ concertó seguros de accidentes indi-

15 Según Tortella (2000, pp. 3-4) la compañía constituida en París en 1911 fundó dos sucursales en España: una contra pedrisco en Madrid y, más tarde, otra contra incendios en Barcelona.

${ }^{16}$ Compañía establecida en Liverpool y Londres como London and Lancasbire Fire and Life en 1861 y reestructurada en 1867 como London and Lancashire Fire. En 1919 se separa la sección de vida creando la London and Lancashire Life (Londres y Escocia). Se especializa en el negocio del seguro de incendio en Gran Bretaña, EEUU y en ultramar desde 1862 y desde 1899 negocia también con el seguro de accidentes. Cockerell y Green (1994, p. 150). 
viduales desde 1916, la Norwich Union Fire ${ }^{17}$ transportes desde 1921 y accidentes individuales desde 1926 y la The Northern Assurance Company Limited ${ }^{18}$ creó secciones de accidentes de trabajo e individuales desde 1923.

La tercera etapa se dio en la década de 1930, cuando las compañías más reacias a la diversificación finalmente tuvieron que asumir este cambio y cuando las entidades de nueva creación aparecían ya como compañías generales de seguros. Una de las empresas más antiguas del ramo de incendios, La Previsión Española, se mantuvo exclusivamente en este negocio hasta 1937 cuando fue autorizada en el ramo de accidentes de trabajo y accidentes individuales y un año más tarde extendió su actividad a los de vida y transportes, convirtiéndose, de facto, en una compañía de seguros generales. La prueba de que el proceso característico de la etapa anterior se había consolidado está en el hecho de que las nuevas compañías que se fueron creando, a partir de finales de la década de 1920, tales como la Peninsular (fundada en 1929), la Sociedad General Española de Seguros y Reaseguros (1928) y La Unión Española (1928), ya se fundaron como compañías generales de seguros, y de que a partir de los años treinta la razón social de muchas entidades incorporase «compañía general de seguros».

Este proceso de diversificación espontáneo realizado en el mercado de seguros español culminó tras la Guerra Civil española y se vio reforzado oficialmente por la Ley de Seguro Privado de 1954, que permitió a las compañías la extensión de sus actividades a todos los ramos que quisieran sin abonar más depósitos ni fianzas. Con ello se cerró un proceso a través del cual pasarán de dominar el mercado las compañías especializadas a ser dominantes las compañías que diversificaron su actividad (La Estrella, 1959). Sin embargo, faltarán aún décadas para que se racionalicen los seguros de enfermedad y entierros que quedarán al margen de la diversificación y en manos de compañías cuyos gestores no eran, en muchos casos, profesionales del seguro.

17 Creada en Norwich en 1797. Se dedicó al seguro de incendios en Gran Bretaña y ultramar desde 1824 y al seguro general después de 1908. Cockerell y Green (1994, p. 163).

${ }^{18}$ Fundada en Aberdeen como Nortb of Scotland Fire and Life Assurance Company en 1836 y renombrada en 1898 . Se dedica al seguro de incendios y vida en Gran Bretaña y en el extranjero. Cockerell y Green (1994, p. 162). 


\section{LA CARTELIZACIÓN DEL SECTOR}

El incremento de la competencia en el mercado español había conducido a las empresas que aseguraban en el país a decisiones empresariales individuales de ampliación de sus actividades a nuevos ramos. Estas estrategias se convirtieron en un fenómeno generalizado por un proceso de imitación hasta convertir a las sociedades de seguros generales en las protagonistas del negocio. Sin embargo, las estrategias empresariales de tipo particular se complementaron con otras fórmulas explícitas de control formal del mercado, imitando las asociaciones y cárteles creados en los mercados extranjeros, sobre todo el inglés durante el siglo XIX. Las compañías de seguros inglesas ya experimentaron la cartelización desde la década de 1860. Concretamente Westall (1984) analiza como el incremento de la competencia en incendios entre las compañias londinenses y las provinciales condujo a la creación de The Fire Offices Committee (FOC) que estableció un complejo sistema de tarifas según los riesgos y permitió mantener el dominio de las compañías más fuertes. Posteriormente se llegaron a acuerdos similares en el seguro de accidentes con la creación de Accident Offices Committee en 1894 y Accident Offices Association en $1906^{19}$.

En España durante el siglo XIX encontramos algunos precedentes sobre acuerdos colusivos en los ramos tradicionales más competitivos, tales como el de incendios. A mediados de la centuria se creó el Comité Central de Compañias de Seguros contra Incendios, que perduró hasta 1877. Según Maestro (1993), el objetivo de este comité, a imitación del inglés, era establecer tarifas y vigilar su cumplimiento por parte de las compañías firmantes del acuerdo. Cinco años más tarde se constituyó el Sindicato General de Compañias con dos comités, en Madrid y Barcelona, que mantenía los mismos objetivos. El sindicato estaba integrado por las principales compañías autorizadas en el ramo de incendios: La Urbana, La Unión y el Fénix Español, La Paternal, La Catalana, El Sol, El Águila, El Fénix Francés, El Mundo, La Unión Francesa y El Progreso Nacional. Constan igualmente precedentes de cárteles en otro de los ramos tradicionales, el de los seguros marítimos. Existió un acuerdo explícito firmado en Barcelona a finales del siglo XIX impulsado por las compañías de seguros marítimos Naviera, Aseguradora, Cabotaje y Salvadora, que en 1868 establecieron un Comité de Seguros maritimos para formular pólizas y llegar a acuerdos sobre tarifas en este tipo de seguros (Matilla y Frax, 1996).

${ }^{19}$ Westall (1998, pp. 77-90), Pearson (1991 y 1993), y Cockerell y Green (1994, pp. 212-213). 
A principios del siglo $\mathrm{Xx}$, tras la aprobación de la Ley de 1908 y el incremento de las compañías autorizadas en los ramos de incendios y marítimos (al margen de esta normativa) renacieron las iniciativas en este campo, intentando controlar la guerra de tarifas desatada entre las entidades que operaban en estos ramos y que se relatan en las revistas especializadas de la época. El ramo de incendios presentaba en 1912 el número más elevado de entidades aseguradoras de todos los ramos con la presencia de sociedades extranjeras en un porcentaje del 54 por 100. Por ello, el 18 de noviembre de 1912 se estableció un Convenio de Compañias de seguros contra incendios para Cataluña y Baleares integrado por las más importantes compañías nacionales y extranjeras y dirigido por los representantes de Aurora, La Urbana, Norwich Union y L'Abeille. Desde su fundación en 1912 hasta la Guerra Civil este convenio estableció tarifas que iba actualizando y ampliando según los riesgos. En 1931 se encontraban adheridas al convenio 63 entidades aseguradoras de las que 25 son españolas y 38 extranjeras ( 18 inglesas, 11 francesas y 9 de diversa nacionalidad), creciendo hasta 66 en 1933 (40 extranjeras y sólo 26 españolas). A lo largo de casi tres décadas se llegó a acuerdos sobre los problemas que afectaban al ramo en este territorio como la competencia de las mutualidades o los porcentajes para corredores y agentes que se estableció en un tipo máximo del 20 por 100 .

Sin embargo, este cártel funcionaba únicamente en el ámbito geográfico citado. Por iniciativa de la empresa que lideraba el ranking del ramo - La Unión y el Fénix Español - y con el beneplácito de las principales compañias inglesas en 1918 se creó en Madrid el Comité Español de Aseguradores de Incendios. El Comité formaba parte de la estrategia de Francisco Setuaín, su director desde 1908, para la expansión de la compañía. Según una historia de la empresa, «se realizan los proyectos de tratados con varias compañias inglesas, y a la vuelta de un viaje a París, trae redactado el proyecto para establecer en España el Sindicato General de Seguros en el Ramo de incendios». (La Unión y el Fénix Español, 1946). Para que pudiera hacerse realidad tuvo que contar con las compañias extranjeras mayoritarias en el ramo. En una reunión celebrada los días 11 y 12 de noviembre de 1918 se presentó el proyecto de Reglamento. En el nuevo cártel domiciliado en Madrid se integraron las compañías que formaban parte del convenio para Cataluña y Baleares. Sin embargo, como hemos visto anteriormente, esto no supuso su disolución, sino que siguió funcionando. La junta directiva del Comité Español de Aseguradores de Incendios estaba formada por el propio Francisco Setuaín, José María de Delás - director de La Catalana-y Regino Álvarez 
Santullano - director de La Estrella-. En 1924 esta agrupación pasó a denominarse Sindicato General de Compañias de Seguros contra incendios que operan en España. Según Maestro (1993), los promotores de esta transformación fueron Francisco Setuaín, Agustín Pons - director en España de The Northern, además de miembro del Fire Office's Committee- y Charles Gibert -miembro del Syndicat Étranger de París-. La disolución del convenio, denominado de los Cuarenta y cuatro riesgos, y la constitución del Sindicato General se produjo en una asamblea celebrada el 22 de febrero de 1924. La influencia de las compañías extranjeras en el nuevo cártel, sobre todo británicas y francesas, a través de los impulsores antes citados, quedó reflejadado en un capítulo introductorio de sus estatutos en el que se prohibía la incorporación al sindicato de las compañías británicas que no tuvieran la autorización del Fire Office's Committe (foreign) ni de ninguna compañía francesa sin la autorización del Syndicat Étranger. Tampoco se admitían mutuas ni sociedades que actuaran como mutuas. Esta asociación continuó su actividad durante la década de 1930 interviniendo en todos aquellos aspectos relacionados con el ramo y, sobre todo, estableciendo acuerdos sobre tarifas y denunciando aquellas prácticas de las compañías asociadas que fueran en contra de los acuerdos del Sindicato. En 1935 todas las compañías nacionales y extranjeras instaladas en España, excepto una que no hemos podido identificar, formaban parte del sindicato.

También el seguro de transportes presentaba una fuerte competencia, no tanto por la establecida entre las compañías instaladas en España, sino por la costumbre de cubrir en plazas extranjeras los seguros de las flotas y buques españoles ${ }^{20}$. Existían varios procedimientos por los que compañías no inscritas en España y también la Lloyds inglesa captaban los negocios de seguros marítimos de mercancías. Una forma era emitir pólizas flotantes a través de sus sedes en el extranjero sobre mercancías a comerciantes españoles para amparar seguros de transporte en general. Otro mecanismo era a través de los brokers ingleses ${ }^{21}$ domiciliados en Londres que, de acuerdo con un representante o corresponsal de España, abrian a su nombre una póliza para asegurar en la Lloyds ${ }^{22}$ todas aquellas expediciones que podían obtener de la clientela de España. Estas prácticas permitían unas primas más bajas, puesto que no debían pagar impuestos ni el asegurado ni el intermediario ni las compañías, y, por tanto, per-

\footnotetext{
20 «La clandestinidad en el Seguro de Transporte», El Eco del Seguro, junio de 1935.

${ }^{21}$ Un estudio sobre un broker de la Lloyds, Hogg Robinson, en Chapman (1984).

22 Sobre las compañias de transporte y la competencia de la Lloyds véase «Las compañías españolas de seguros», El Eco del Seguro, abril de 1926.
} 
judicaban a las sociedades inscritas en España. A pesar de la competencia del seguro exterior, las compañías en España habían creado en 1913 el Comité Español de Aseguradores Marítimos con los objetivos de reducir los efectos de la competencia y de la guerra de tarifas en el sector. En 1930 el ramo está plenamente sindicado de forma que casi la totalidad de las compañías que operaban en él formaban parte de este comité ${ }^{23}$.

Son muchos los artículos que aparecen en las revistas especializadas a favor de los cárteles frente al régimen libre en la actuación de las compañías por ramos. La sindicación fue impulsada por las grandes compañías españolas y extranjeras en todo el período estudiado. En las revistas de seguros aparecen constantes referencias a lo positivo de estos acuerdos. En 1928 Salvador Coca publicaba un artículo en el que apoyaba el agrupamiento y el pacto de las compañías para igualar las primas, condiciones de pólizas y el máximo de remuneración a los agentes ${ }^{24}$. Este sistema «consolida las carteras y estabiliza los ingresos, permitiendo que éstos se acomoden siempre a las alternativas de un mayor encarecimiento de los gastos generales, de la agravación de los riesgos, etc.». Sin embargo, según el autor, existían aún mecanismos que quedaban al margen de la sindicación, como la remuneración a subdirectores o delegados, entre otros, que era peculiar en cada compañía y la ampliación de beneficios de los contratos en lo no expresamente legislado. Estos dos elementos de la gestión de las compañías era suficiente para que se produjeran grandes desequilibrios perturbando el objetivo de la sindicación: el afianzamiento de las carteras y que las primas fueran remuneradoras. Además, ante la competencia desatada, muchas compañías sindicadas se atrevían a contratar a primas inferiores de las pactadas, a establecer reglas y condiciones prohibidas y a ceder comisiones o bonificaciones a los asegurados. Por tanto, parece que no existió una sindicación pura, sino que las infracciones y las prácticas alternativas dieron continuidad a la competencia en el sector. Otro de los grandes defensores de la sindicación fue Rafael Iparraguirre --director de La Unión y el Fénix entre 1925 y 1935-, quien en los años treinta publicó un artículo sobre la sindicación en las compañías de seguros en el que establecía los tres fines esenciales de la sindicación: primero, la defensa general de las compañías en materia legislativa, contributiva, etc.; segundo, regulación de primas, comisiones y condiciones de contrato; y

23 «Comité de Aseguradores marítimos», El Eco del Seguro, septiembre de 1930, p. 167. En el artículo se hace referencia a que el comité en estos momentos estudiaba implantar en España algunos acuerdos establecidos por la Joint Hull Committee.

${ }_{24}$ «Régimen libre y régimen de sindicación», Anuario Español de Seguros, 1928. 
tercero, la confección de estadísticas españolas de siniestros aleatorios. En el texto se insistía en luchar contra las prácticas que afectaban a la cartelización, sobre todo las comisiones que dejaban poco rastro y, por tanto, permitían a las compañías prácticas competitivas que atentaban contra los principios de la sindicación.

La cartelización fue considerada, en general, como la forma más positiva de luchar contra la competencia y la guerra de tarifas existente en algunos ramos. Incluso en aquellos de menor entidad se propugnaba la sindicación para atacar los males del negocio. Es el caso del ramo de cristales en el que a pesar de las escasas compañías que operaban presentaba algunos problemas como la tarifación del riesgo que podrian solucionarse constituyendo un convenio a imitación de las compañías del ramo de incendio ${ }^{25}$. En la década de 1930 los ramos de mayor entidad y con mayor competencia, a excepción del ramo de vida, se encuentran plenamente sindicados influenciados por el modelo británico y, en menor medida, francés debido posiblemente a la importancia de las compañías extranjeras en estos ramos.

\section{CONCLUSIONES}

Sobre la base heredada del siglo XIX, entre 1912 y 1935 el negocio de los seguros en España se modernizó y racionalizó en gran parte debido a la introducción de normas que obligaron a las compañías a establecer un capital social y unas reservas mínimas, en especial las compañías de seguro de vida, a presentar a inspección las pólizas y la publicidad y, en resumen, a cumplir toda una serie de requisitos que garantizaran los derechos de los asegurados.

Paralelamente al creciente control oficial, el negocio del seguro creció en los ramos de vida, incendios, transportes y accidentes mientras entraban en decadencia algunos ramos propios del siglo anterior como los seguros bajo el sistema de las tontinas o la fórmula chatelusiana, el seguro sobre ganado y permanecían estancados ramos como los seguros de enfermedad o cristales. Las compañías, tanto españolas como extranjeras, que aseguraban a principios de siglo, algunas de ellas creadas en el siglo XIX, eran sociedades especializadas en un solo ramo. En los más tradicionales, incen-

25 «No es extraño, pues, que el articulista, aun cuando poco avezado en estas lides aseguradoras, se atreva a poner pica en Flandes y cantar las excelencias del convenio de Compañias de Incendios que ban tenido el valor de atacar el mal que las devoraba y acabar de una vez con la desdichada competencia», El Eco del Seguro, febrero de 1926, pp. 7-8. 
dios y transportes, a las numerosas entidades que ya participaban en el mercado se unieron otras en las primeras décadas del siglo $\mathrm{XX}$ que obstaculizaron el crecimiento de las primas medias por empresa e incrementaron la competencia. Ante esta situación las entidades más importantes, tanto españolas como extranjeras, impulsaron dos estrategias para impedir que con la guerra de tarifas disminuyeran sus beneficios: la diversificación del negocio y la sindicación o cartelización.

La diversificación se experimentó a partir de finales de la década de 1910, consolidándose en la década siguiente. En estos años las principales aseguradoras fueron ampliando sus actividades desde su especialización hacia el resto de ramos principales. De esta forma las entidades que operaban en España se fueron convirtiendo en compañías generales de seguros. Las sociedades extranjeras también participaron diversificando sus operaciones en España, aunque posiblemente ligadas al mismo fenómeno que ya había experimentado su casa matriz. La ampliación de actividades de las empresas de seguros se difundió miméticamente entre las aseguradoras en las décadas anteriores a la Guerra Civil. Posteriormente, este proceso fue impulsado por el Estado, de forma oficial con la nueva Ley de Seguros de 1954 que permitió a las entidades de seguro la ampliación a todos los ramos que deseasen sin ningún coste.

En los ramos más competitivos como el seguro de incendios y de transporte se crearon cárteles a imitación de los Comittees ingleses o la Syndicats franceses con el objetivo de establecer acuerdos formales para el control de mercado, fijar tarifas y prohibir determinadas prácticas que gravaban la competencia del ramo. Estos sindicatos o comités fueron impulsados por las compañías españolas y extranjeras con mayor volumen de negocio intentando limitar la actuación de aquellas entidades no sindicadas. Esta práctica se interrumpió con la guerra y la creación por parte de la dictadura franquista del Sindicato Nacional del Seguro, fruto de ese nuevo concepto de un Estado Nacional Sindicalista, en el que tuvieron que integrarse obligatoriamente todas las asociaciones y sindicatos creados en la etapa anterior.

Esta dualidad de procesos benefició a las grandes compañías que consolidaron su posición ya inicialmente preeminente. Éste fue el caso de La Unión y el Fénix Español que desde su creación en el siglo XIX operaba en el ramo de incendios y seguros marítimos y que lideró la sindicación de ambos ramos además de encontrarse entre las primeras compañías que acometieron su diversificación. Este liderazgo en ambos procesos dio como resultado que en 1940 ocupara el primer puesto en el ranking de primas cobradas por todas las compañías que aseguraban en España. Su posición 
hegemónica era fruto de ocupar la tercera posición en el ranking en el ramo de vida, la primera en el de incendios, la segunda en el seguro de accidentes de trabajo, la quinta en el ramo de accidentes individuales y de responsabilidad civil y, finalmente, el segundo lugar en el ranking del ramo de transportes. Los procesos de diversificación y cartelización que caracterizan el crecimiento de las entidades aseguradoras en España fueron acompañados de otras transformaciones en la gestión, de acuerdos colusivos y de la introducción de la banca en el accionariado de las entidades. Estos aspectos no han sido, de momento, objeto de estudios por lo que cuando se aborden nos aportarán un mayor conocimiento del sector.

\section{BIBLIOGRAFÍA}

Bathmonde Magro, A. (1981): El Horizonte Económico de la Burguesia Isabelina: Madrid 1856-1866, Madrid, Universidad Complutense de Madrid.

BiBilONI, J., y PONS, J. (1999): «El desarrollo de las mutualidades patronales de accidentes de trabajo en España. El mercado balear: entre la competencia y la colusión (1920-1940)», Revista de Historia Industrial, núm. 15, pp. 83-104.

Burgaz, J., y Pérez-Morales, M. (1996): 1902-1992. 90 años de seguros agrarios en España, Madrid, Ministerio de Agricultura, Pesca y Alimentación.

BuTT, J. (1984): «Life assurance in war and depression: the Standard Life Assurance Company and its environment, 1914-1939», en O. M. Westall (ed.), The bistorian and the business of insurance, Manchester, pp. 155-172.

Castillo, S. (ed.) (1994): Solidaridad desde abajo, Madrid, UGT - Centro de Estudios Históricos y Confederación Nacional de Mutualidades de Previsión.

Catalana OCCIDENTE (1989): 125 años de CATALANA OCCIDENTE, Barcelona.

Chapman, S. D. (1984): «Hogg Robinson: the rise of a Lloyd's broker», O. M. WESTALl (ed.), The historian and the business of insurance, Manchester, pp. 173-189.

Cockerell, H. A. L, y Green, E. (1994): The British Insurance Business. A Guide to its History \& records, London.

Cuesta Bustillo, J. (1988): Los seguros sociales en la España del siglo XX. Hacia los seguros sociales obligatorios, Madrid, Ministerio de Trabajo y Seguridad Social.

DinsDale, Ph. D. (1954): History of Accident Insurance in Great Britain, London. Estrella (LA) (1959): Medio Siglo del Seguro Privado Español, Madrid.

FANFANI, T. (1998): «L'assurance-vie en Italie entre iniciative privée et monopole (XIXe siècle-début XXe siècle)», en C. E. NúNEz (ed.), Insurance in industrial societies: economic role, agents and market from $18^{\text {th }}$ century to today, Sevilla, pp. 31-55.

FisHBACK y KanTOR (1996): «The Durable Experiment: State Insurance of Workers' Compensation Risk in the Early Twentieth Century», The journal of Economic History, vol. 56, núm. 4, pp. 809-836. 
- (1998): «The Political Economy of Workers' Compensation Benefit levels, 1910-1930», Explorations in Economic History, núm. 35, pp. 109-139.

FraX, E., y Matilla, M. J. (1996): «Los seguros en España: 1830-1934», Revista de Historia Económica, año XIV, núm. 1, pp. 183-203.

- (1998): «La evolución del sector seguros en Francia y España. 1800-1936», C. E. NúNEZ (ed.), Insurance in industrial societies: economic role, agents and market from $18^{\text {th }}$ century to today, Sevilla, pp. 31-44.

Gallegos Romero, J. E. (1967): Dirección de las inversiones en las compañias de seguros, Madrid, Riesgo y Seguro.

Gibaud, B. (1997): «La formation de la personnalité du mutualisme français (1850-1930)», Pré-session del XII Congrés international d'Histoire économique, 30-31 de mayo de 1997 en París.

GonzAlez-Bueno Lillo, G. (1986): «El sistema español de seguros agrarios», Hacienda Pública Española, I, pp. 173-185.

Hautcoeur, P. C., y Verley, P. (1998): «Les plaçements des compagnies françaises d'assurances sur la vie (1860-1939)», en C. E. NúNEZ (ed.), Insurance in industrial societies: economic role, agents and market from $18^{\text {th }}$ century to today, Sevilla, pp. 159-175.

JENKINS, D. T. (1984): «The practice of insurance against fire, 1750-1840, and historical research», en O. M. WeSTALl (ed.), The bistorian and the business of insurance, Manchester, pp. 9-38.

KELlER, M. (1963): The Life insurance enterprise, 1885-1910. Study in the limits of corporate Power, Cambridge, The Belknap Press of Harvard University Press.

La Unión y el FéndX Español (1946): 80 Aniversario, 1864-1944, Madrid.

Maestro, M. (1991): Madrid, capital aseguradora de España, tomo III de Historia del Seguro Español, Madrid.

- (1993): Formación del Mercado Español de Seguros, 1908-1960, Madrid.

Montero, F., y Martínez Quintero, M. ${ }^{a}$ E. (1988): Origenes y antecedentes de la Previsión Social, Madrid, Ministerio de Trabajo y Seguridad Social.

PEARSON, R. (1991): «Collective diversification: Manchester cotton merchants and the insurance business in the early nineteenth century», Business History Review, núm. 65 , pp. 379-414.

- (1993): «Taking risks and containing competition: diversification and oligopoly in the fire insurance markets of the north of England during the early nineteenth century», Economic History Review, XIVI, I, pp. 39-64.

Pons Pons, J. (1998): El sector seguros en Baleares. Empresas y empresarios en los siglos XIX y XX, Palma de Mallorca, El Tall.

- (2000): «Jesús Serra Santamans», en Eugenio Torres (ed.), Los 100 empresarios españoles del siglo XX, Madrid, Lid Editorial, pp. 451-455.

- (2001): «Las entidades aseguradoras y la canalización del ahorro en España, 1908-1940», Actas Congreso de la Asociación de Historia Económica, Zaragoza.

- (2002a): Las Estrategias de crecimiento de las compañias de seguro en España, 1900-1940, Documento de trabajo de la Fundación Empresa Pública. Programa de Historia Económica, núm. 2002/1.

- (2002b): «La aportación de las compañias de seguros de vida norteamericanas en la formación del mercado español, 1909-1940», Seminario sobre La Ame- 
ricanización en España: 50 años de influencia económica y social, celebrado en la Universidad Complutense de Madrid, 12-13 de septiembre de 2002.

Rodriguez Ocañ, E. (1990): «La asistencia médica colectiva en España, hasta 1936», en Historia de la Acción Social pública en España, Madrid, Beneficencia y Previsión, Ministerio de Trabajo y Seguridad Social, pp. 321-359.

Romero Magallaes, J. (1998): «Les premiers projects de municipalisation et nationalisation des assurances au Portugal au debut du XXè siècle», en C. E. NúNEZ (ed.), Insurance in industrial societies: economic role, agents and market from $18^{\text {th }}$ century to today, Sevilla, pp. 45-55.

RYAN, R. (1984): «The Norwich Union and the British fire insurance market in the early nineteenth century», O. M. WESTALL (ed.), The historian and the business of insurance, Manchester, pp. 39-73.

Samaniego, M. (1988): La unificación de los seguros sociales a debate: La segunda República, Madrid, Ministerio de Trabajo y Seguridad Social.

Sudria, C. (1998): «Las Cajas de Ahorros en los orígenes del Retiro Obrero (1900-1939), Papeles de Economia Española, núm. 74-75, pp. 323-330.

Supple, B. (1984): «Insurance in British history», en O. M. Westall (ed.), The bistorian and the business of insurance, Manchester, pp. 1-8.

TorTella, T. (2000): Una guía de fuentes sobre inversiones extranjeras en España (1780-1914), Madrid.

Westall, O. M. (1984): «David and Goliath: The Fire Offices Committee and non tariff competition, 1898-1907», en O. M. WESTALL (ed.), The bistorian and the business of insurance, Manchester, pp. 130-154.

- (1994): «Marketing Strategy and the Competitive structure of British General Insurance, 1720-1980», Business History, 36, 2, pp. 20-46.

- (1997): «Invisible, Visible and "Direct" Hands: an Institutional Interpretation of Organisational Structure and Change in British General Insurance», Business History, 39, 2, pp. 44-66.

- (1998): «Collusion and competition: the framework of enterprise in British general insurance», en C. E. NúNEZ (ed.), Insurance in industrial societies: economic role, agents and market from $18^{\text {th }}$ century to today, Sevilla, pp. 77-90.

\section{FUENTES IMPRESAS}

Anuaric Español de Seguros (1928) (BNE = Biblioteca Nacional de España).

Anuario Estadístico de España (1919) (BNE).

Anuario Guia del Asegurador (1924) (Centro de documentación del INESE = Instituto de Estudios Superiores Financieros y de Seguros).

Boletín de la Inspeción Mercantil y de Seguros (1927-1928) (BNE).

Boletin Oficial de Seguros (1913-1926) (BNE).

Boletín Oficial de Seguros y Aborro (1934-1941) (BNE).

El Eco del Seguro (1926-1933) (INESE).

Revista de Previsión (1929-1934) (INESE).

Revista Los Seguros (1918) (INESE). 